\title{
Thai Finger-Spelling Recognition Using a Cascaded Classifier Based on Histogram of Orientation Gradient Features
}

\author{
Kittasil Silanon \\ Department of Computer Engineering, Faculty of Engineering, Prince of Songkla University, Kathu, Phuket 83120, Thailand \\ Correspondence should be addressed to Kittasil Silanon; kittasil.silanon@gmail.com
}

Received 24 April 2017; Accepted 5 July 2017; Published 6 September 2017

Academic Editor: Fabio La Foresta

Copyright ( 2017 Kittasil Silanon. This is an open access article distributed under the Creative Commons Attribution License, which permits unrestricted use, distribution, and reproduction in any medium, provided the original work is properly cited.

\begin{abstract}
Hand posture recognition is an essential module in applications such as human-computer interaction (HCI), games, and sign language systems, in which performance and robustness are the primary requirements. In this paper, we proposed automatic classification to recognize 21 hand postures that represent letters in Thai finger-spelling based on Histogram of Orientation Gradient (HOG) feature (which is applied with more focus on the information within certain region of the image rather than each single pixel) and Adaptive Boost (i.e., AdaBoost) learning technique to select the best weak classifier and to construct a strong classifier that consists of several weak classifiers to be cascaded in detection architecture. We collected 21 static hand posture images from 10 subjects for testing and training in Thai letters finger-spelling. The parameters for the training process have been adjusted in three experiments, false positive rates (FPR), true positive rates (TPR), and number of training stages (N), to achieve the most suitable training model for each hand posture. All cascaded classifiers are loaded into the system simultaneously to classify different hand postures. A correlation coefficient is computed to distinguish the hand postures that are similar. The system achieves approximately $78 \%$ accuracy on average on all classifier experiments.
\end{abstract}

\section{Introduction}

Sign language is a communication method for deaf or nonvocal people. For a sign language system, there are two main categories: (1) word-level vocabulary signs, which are signs of the hand shape, orientation and movement of the hands, arms, or body, and facial expressions simultaneously to represent word meanings, and (2) finger-spellings, which use only hand shape to spell the letters of the word in a spoken language, representing names, places, technical terms, and so on. However, most deaf and nonvocal persons, especially children, have problems with finger-spelling skills because finger-spelling is used infrequently in daily communication. Therefore, in order to help these people improve their skills, many systems specific to finger-spelling were proposed, for example, the American (ASL) (Dinh et al. [1], Feris et al. [2], Ricco and Tomasi [3], and Mo and Neumann [4]), British (BSL) (Goh and Holden [5]), Australian (Auslan) (Liwicki and Everingham [6]), Chinese (CSL) (Jiangqin and Wen [7] and Teng et al. [8]), and Japanese (JSL) (Fujimura and Liu [9] and Tabata and Kuroda [10]). In this work, we have focused on Thai finger-spelling (ThSL). Saengsri et al. [11] proposed a Thai letter finger-spelling by using the data glove, a motion tracker, and Neural Network theory to improve the accuracy of the system. Kanjanapatmata [12] presented an image recognition method for the Thai letter using a polar orientation histogram of the hand image and an artificial Neural Network. Sakulsujirapa et al. [13] presented an appearance feature lookup table to analyze hand posture patterns for identifying Thai letters in finger-spelling. Sriboonruang et al. [14] proposed a method combining the Zernike moment and wavelet moment to capture a hand's features and also using a fuzzy classification algorithm to classify Thai finger-spelling hand postures. Phitakwinai et al. [15] developed the Thai finger-spelling letters and words of the Thai sign language translation system using the scale-invariant feature transform (SIFT). However, they cannot achieve the critical criteria, such as accuracy, flexibility, and device constraints, and cannot run in real time.

In this paper, we developed automatic classification to recognize 21 hand postures that represent letters in Thai 


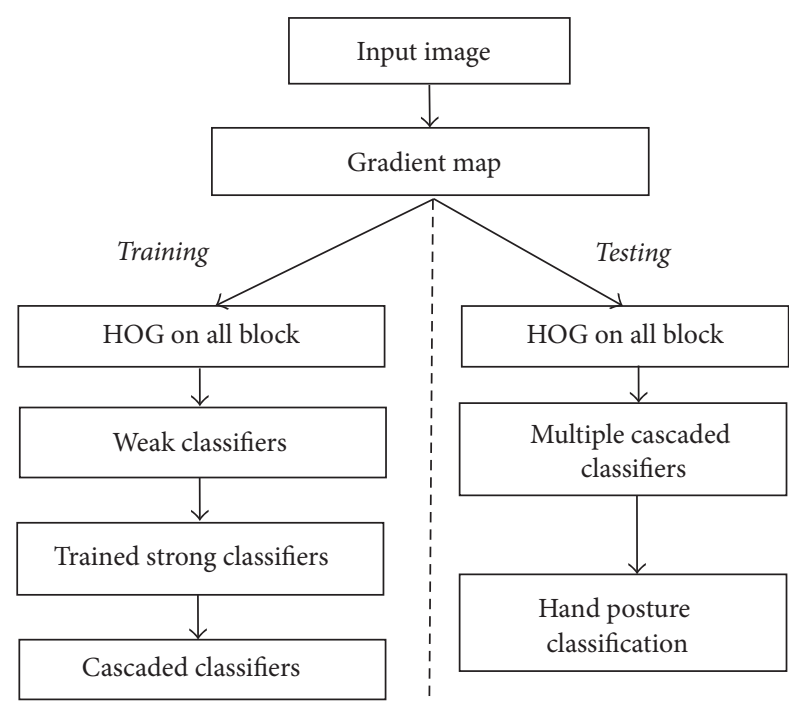

FIGURE 1: Thai letter finger-spelling recognition using HOG.

finger-spelling. In our implementation, an object detection approach based on Histogram of Orientation Gradient (HOG) feature is applied as the main feature of hand postures which focuses more on the information within a certain region of the image rather than each single pixel. A feature is trained to be a weak classifier using a histogram comparison. In order to improve the detection speed, the weak classifiers are trained into strong classifiers by the AdaBoost algorithm, which were finally combined into a cascaded classifier for the detection procedure. The experiment is designed to adjust training parameters, false positive rates (FPR), true positive rates (TPR), and number of training stages $(N)$, to achieve a suitable training model for 21 hand postures. All cascaded classifiers are loaded into the system simultaneously to classify different hand postures. The correlation coefficients are computed between inputs and pattern data to distinguish the hand postures that are similar. The system process in this method is shown in Figure 1.

\section{Proposed Method}

This system recognizes 5 numbers and 16 letters of Thai finger-spelling (Silanon [17]) which use single hand postures. The digits and letters we recognize are the numbers " 1 ," " 2 ," “3," “4," and "5" and letters “อ” (O ang), "บ" (Bo bimai), “ด” (Do dek), “ฟ” (Fo fan), “ห” (Ho hip), “จ” (Cho chan), “ก” (Ko kai), “ล” (Lo ling), “ม” (Mo ma), “น” (No nue), “พ” (Po phan), “ร” (Ro ruea), “ส” (So suea), “ต” (To tao), “ว” (Wo waen), and “ย” (Yo yak). We collected these hand postures from 10 subjects who were asked to stand in front of white background. A web camera is used to capture an image resolution of $640 \times 480$ pixels in laboratory with a light condition. For each hand posture, there are 100 for training and 50 for testing. The 21 hand postures for Thai letter fingerspelling are shown in Figure 2.

Next we collected all hand postures into a dataset for a recognition process. We utilized HOG as the feature descriptor for each hand posture. Let us introduce HOG. The HOG features were used in many papers that address the object detection problem (Dalal and Triggs [18], Li et al. [19], Liu et al. [20], and Zhu et al. [21]). For HOG extraction, the first step of the calculation is the computation of the gradient values. This method requires filtering the gray scale image with the following filter kernels:

$$
\begin{aligned}
& D_{x}=\left[\begin{array}{lll}
-1 & 0 & 1
\end{array}\right], \\
& D_{y}=\left[\begin{array}{lll}
-1 & 0 & 1
\end{array}\right]^{T} .
\end{aligned}
$$

Therefore, given an image I, we obtain the $x$ and $y$ derivatives using a convolution operation:

$$
\begin{aligned}
& I_{x}(r, c)=I(r, c+1)-I(r, c-1), \\
& I_{y}(r, c)=I(r-1, c)-I(r+1, c) .
\end{aligned}
$$

The orientation of the gradient is then transformed to polar coordinates $(\theta)$, with the magnitude of the gradient $|G|$ :

$$
\begin{gathered}
\theta=\tan ^{-1} \frac{I_{y}}{I_{x}}, \\
|G|=\sqrt{I_{x}^{2}+I_{y}^{2}} .
\end{gathered}
$$

The image window is divided into a small spatial cell of size of $8 \times 8$ pixels. The cells are rectangular, and the histogram channels are spread over 0 to $180^{\circ}$, which determine 9 bins. We group the $2 \times 2$ cells into single block feature $(\mathbf{b})$ and normalize the block feature to reduce the effect of change in contrast between images of the same object by its Euclidean norm:

$$
\mathbf{b}=\frac{\mathbf{b}}{\|\mathbf{b}\|^{2}+\varepsilon} .
$$

In this expression, $\varepsilon$ is a small positive constant that prevents a division by zero. A dimension feature of each block is determined by the number of orientation bins in each cell. Therefore, there are 36 dimensions for a block feature. Figure 3 showed the process of HOG feature calculations.

The second step is to study the construction of the weak classifier $\left(h_{j}\right)$ for hand postures. We estimate the distance between the histogram of an input feature $\left(x_{j}\right)$ and a model histogram $m_{j}$. The model calculated the average histogram between all training positive examples. For each histogram of the feature set, we have its corresponding model $m_{j}$. We define the weak classifier as a binary function $h_{j}(x)$ :

$$
h_{j}(x)= \begin{cases}1 & \text { if } d\left(x_{j}, m_{j}\right)<\theta_{j} \\ 0 & \text { otherwise }\end{cases}
$$

where $d\left(x_{j}, m_{j}\right)$ is the histogram comparison (Negri et al. [22]) between the feature $x_{j}$ and the model $m_{j}$ and $\theta_{j}$ is the feature threshold. In practice, no single weak classifier can identify the object with high accuracy. We used the AdaBoost learning algorithm (Chen and Georganas [23], 


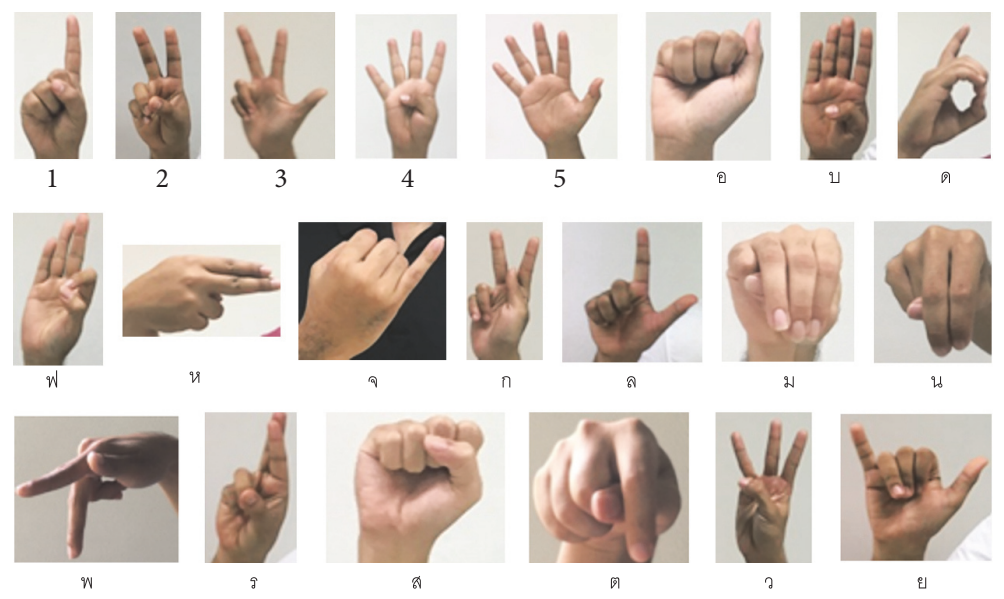

FIgURE 2: The 21 example hand postures.

(1) Calculate image gradient magnitude and orientation for each pixel

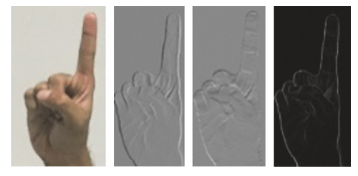

$$
\begin{aligned}
& I_{x}=I(r, c+1)-I(r, c-1) \\
& I_{y}=I(r-1, c)-I(r, c+1) \\
& |G|=\left(I_{x}+I_{y}\right)^{1 / 2} \\
& \theta=\tan ^{-1}\left(I_{y} / I_{x}\right)
\end{aligned}
$$

(2) Accumulate weight vote into orientation bins over spatial cells

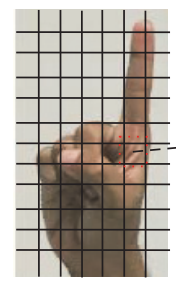

Input

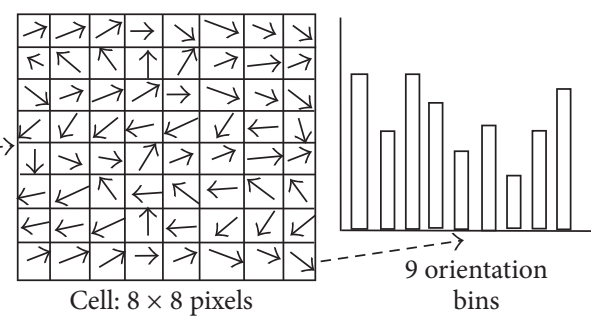

(3) Group cells and normalize block feature

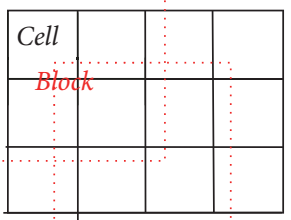

(i) Dimension of each block:

$2 \times 2 \times 9=36 D$

(ii) Norm: $\mathbf{b}=\mathbf{b} /\left(\|\mathbf{b}\|^{2}+\varepsilon\right)$

FIGURE 3: HOG feature calculation.

Pavani et al. [24], and Viola and Jones [25]), which can improve the accuracy detection. Now let us review the AdaBoost algorithm. The algorithm takes as input a set of $M$ training samples, labeled as negatives (0) or positives (1): $\left\{\left(x_{1}, y_{1}\right) \cdots\left(x_{N}, y_{N}\right)\right\}$, where $y_{i}$ is the label of a certain instance $x_{i}$, as shown in Figure 4 .

Then a group of classifiers is tested from the set of samples and the best weak classifier, according to a minimum error, is chosen. Finally, the algorithm computes the parameter $\alpha$ associated with the chosen weak classifier, which measures the importance of the weak classifier's contribution to the final strong classifier. The process is repeated $T$ times, extracting a new weak classifier per iteration. Thus the weak classifier and the corresponding weight are determined through the boosting procedure. The prediction of a strong classifier for the binary classification problem has the following form:

$$
H(\mathbf{x})= \begin{cases}1, \text { object } & \sum_{i=1}^{k} \alpha_{i} h_{i}(\mathbf{x}) \geq \frac{1}{2} \sum_{i=1}^{k} \alpha_{i} \\ 0, \text { clutter } & \text { otherwise. }\end{cases}
$$

The pseudocode of AdaBoost algorithm adapted to the object detection problem is shown in Pseudocode 1.

For object detection, a cascaded classifier is built which consists of serially connected nodes labeling a test image as either object or clutter. Each node contains a boosted set of weak classifiers. In Figure 5, the third node of the cascaded classifier is expanded to show the $k$ weak classifiers presented inside it. A given test image is scanned at all positions and scaled by the cascaded classifier. When an image subregion $\mathbf{x}$ is put to a node, it is classified by all the weak classifiers presented in the node, and the weighted average of their decisions is calculated as the final decision of that node. An image subregion is labeled as an object when it is identified as object by $M$ nodes of the cascade (see Figure 5). On the other hand, if a subregion is labeled as a clutter by any node, it will not be processed by the successive nodes. Thus, a detection system based on cascaded classifier architecture will be fast in scanning the entire test image.

\section{Experiments}

The experiments are conducted in the laboratory with controlled light conditions. The positive training set images were collected as 100 original samples for each hand posture. However, we can also generate more positive samples from existing ones by varying the brightness or the contrast. Thus, we have a set of 500 training images for each hand posture. The negative samples come from 17,000 images without a hand posture. The cascaded training process involves two types of trade-offs (1) the number of stages $(N)$ and (2) 
(i) Given images $\left(x_{i}, y_{i}\right)$ where $y_{i}=0,1$ for negative and positive examples.

(ii) Initialize weight $w_{1, i}=1 / 2 m, 1 / 2 l$ for $y_{i}=0,1$ respectively,

where $m$ and $l$ are the number of negatives and positive.

(iii) Set of weak classifier $h_{j}$

(iv) Initial True Positive Rate and the False Positive Rate

For $t=1, \ldots, T$ :

(1) Normalize the weights, $w_{t, i} \leftarrow w_{t, i} / \sum_{j=1}^{n} w_{t, j}$

(2) the error of the weak classifier: $\varepsilon_{j}=\sum_{i} w_{i}\left|h_{j}\left(x_{i}\right)-y_{i}\right|$

(3) Choose the classifier, $h_{j}$, with lowest error

(4) Set $\beta_{t}=\varepsilon_{t} /\left(1-\varepsilon_{t}\right)$ and $\alpha_{t}=\log \left(1 / \beta_{t}\right)$

Update weights: $w_{t+1, i}=w_{t, i} \beta_{t}^{1-\left|h_{j}\left(x_{i}\right)-y_{i}\right|}$

(v) The final strong classifier is: $H(x)=\sum_{i=1}^{K} \alpha_{i} h_{i}(x)$

Pseudocode 1: The AdaBoost algorithm.

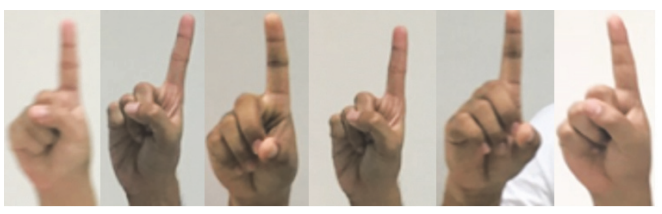

(a)

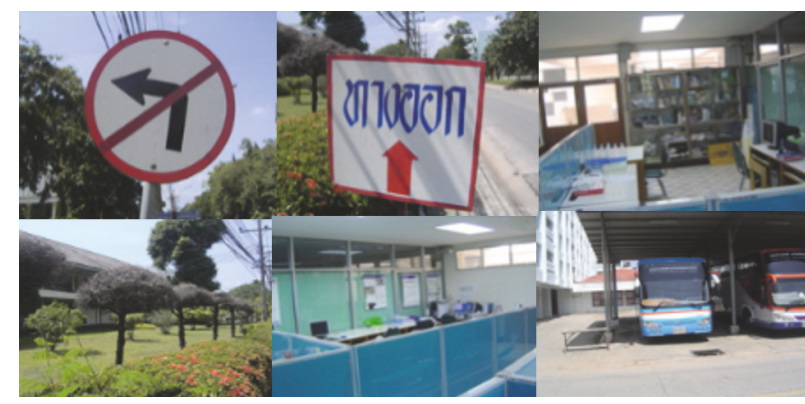

(b)

Figure 4: (a) Positive samples and (b) negative samples.
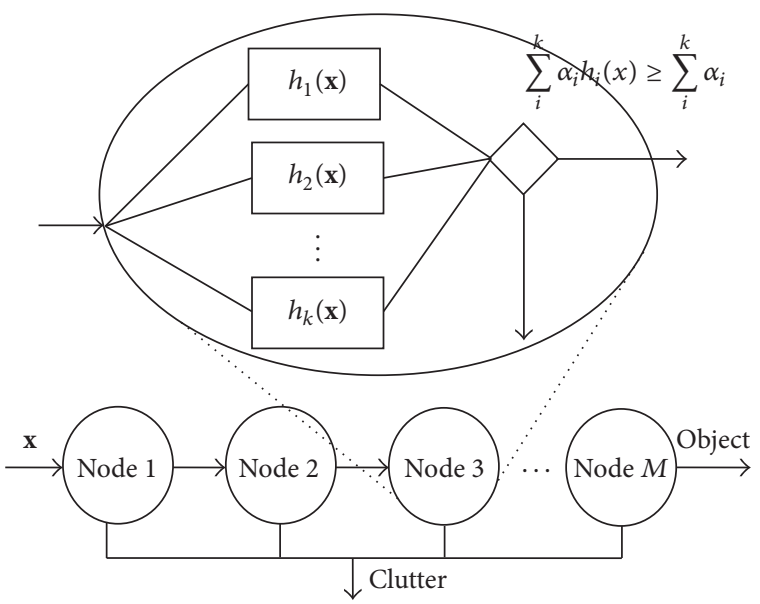

FIGURE 5: The structure of cascaded classifier.

the threshold of true positive rate (TPR) and false positive rate (FPR) of each stage to achieve higher detection and a lower false positive rate. Unfortunately, finding this optimum is a tremendously difficult problem. In practice, a simple hypothesis structure is used to produce an effective classifier, which is highly efficient. Each stage in the cascade reduces the false positive rate and increases the true positive rate. A classifier is trained by adding a number of stages until the target for false positive rate and detection rate is met (these rates are determined by testing the detector on a testing set). A target is selected for the maximum reduction in false positive rate while maintaining the minimum decrease in detection.

To test a hypothesis, there are three experiments based on testing different parameters to determine better performance of the classifier. We divide the experiment into three parts, that is, training with FPR, training with TPR, and training with $N$. In the first experiment, we tested the performance of correct classification with different FPR: the fraction of negative training samples incorrectly classified as positive samples or values in range $(0,1]$. This value is varied from 0.05 to 0.5 in step of 0.05 . Other training parameters are fixed; for example, TPR is $0.995, N$ is 5 , and training size is $32 \times 32$ pixels. To evaluate the performance of the training classifier, 50 images with a similar background and light condition (which are not used as training samples) for each hand posture class are tested. Each image has a resolution of $640 \times 480$ pixels. Table 1 shows the performance of 21 trained classifiers for all test images. Figure 6 shows some of the detection results of the " $n$ " (Ko kai) hand posture from each FPR value. From Table 1, outcomes are called "Hit" and "Miss" and "False" detection. The "Hit" detection is that hand posture is presented: the classification model must decide whether a hand posture is presented. The "Miss" detection is that hand posture is presented: the classification model decides otherwise. The "False" detection is an error in the evaluation process, in which a tested image is mistakenly found to be detected. 


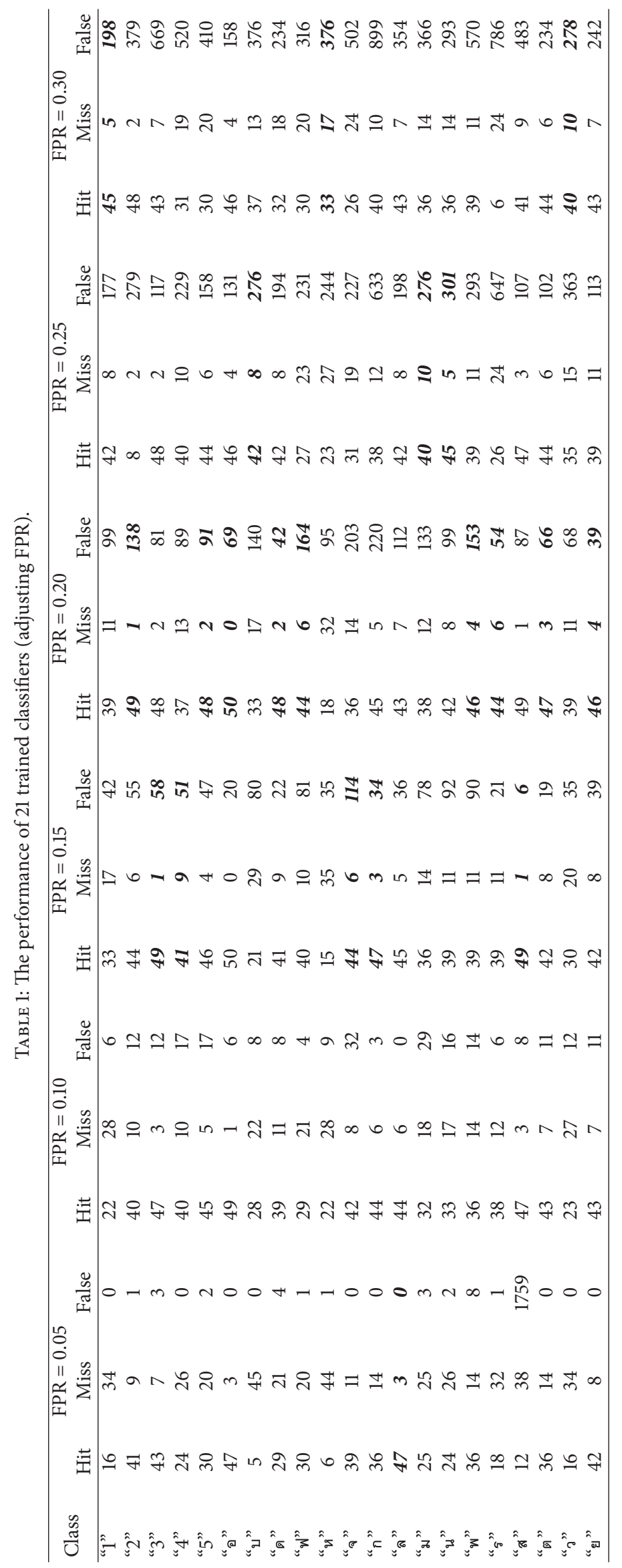




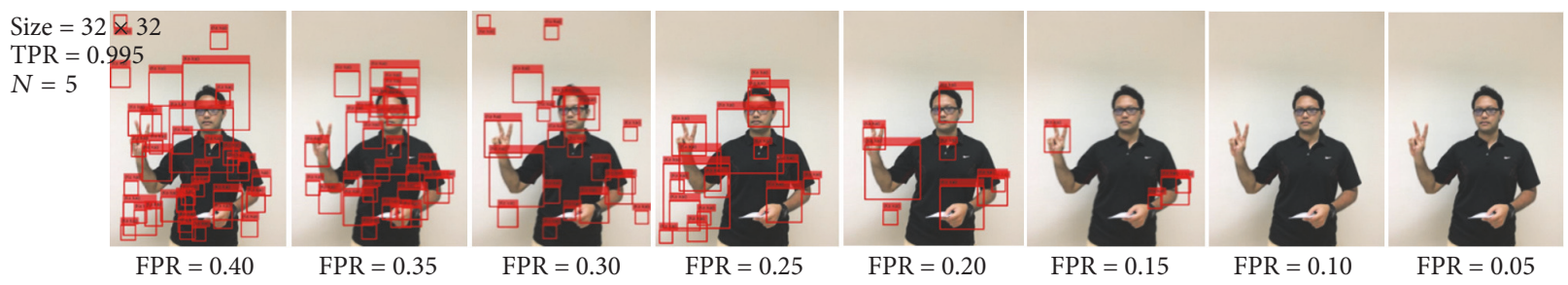

Figure 6: Result for “ $\mathrm{n}$ ” (Ko kai) hand posture (adjusting FPR).

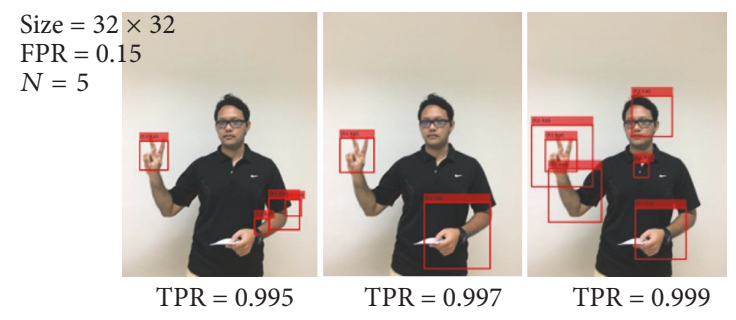

FIGURE 7: Result for “ $\cap$ ” (Ko kai) hand posture (adjusting TPR).

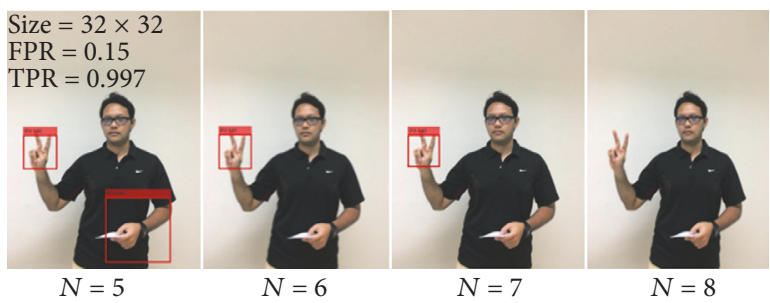

Figure 8: Result for “ $n$ ” (Ko kai) hand posture (adjusting $N$ ).
We heuristically find which value of FPR optimizes the performance of our classifier model. This value is varied experimentally based on each hand posture to achieve the best result. From experiment, the most suitable value of FPR for model is ranging from 0.05 to 0.30 . The value of this parameter, for each hand posture class, is chosen from the case of maximum "Hit" detection (which is italicized bold in Table 1). For example, the hands posture class of " $n$ " (Ko kai) with a FPR of 0.15 is selected because it provides the maximum "Hit" detection result. By analyzing the experimental result carefully, we found that lower value for FPR can achieve less "False" detection. Nevertheless, results in "Miss" detection and "False" detection are still not suitable for use in real-time applications.

To reduce the probability of "Miss" and "False" detections, the second experiment is implemented to increase TPR: the fraction of correctly classified positive training samples or values in range $(0,1]$. This parameter was varied as 0.995 (1st experiment), 0.997, and 0.999, respectively. $N$ is still 5 . FPR for each hand posture class is also selected from the first experiment. Table 2 shows the performance of 21 trained classifiers with different TPR. According to this experiment, the results of some hand posture classes have improved (which are italicized bold in Table 2). For instance, for the class of " $n$ " (Ko kai), TPR of 0.997 is selected. However, this value does not affect to the "Miss" detection; but the "False" detection impact starts to occur as it has decreased slightly. A high value of the TPR results in a greater number of correct detections. However, it increases the training and detection times. The classification model is chosen from maximum "Hit" detection in each hand posture class. Although the classification model has improved, the "False" detection is still high. Figure 7 shows some of detection results of the " $n$ " (Ko kai) hand posture from each TPR value. To reduce the number of "False" detections, the third experiment is implemented by increasing the number of $N$. The 5th, 6th, 7th, 8th, 9th, and 10th stages were trained. FPR and TPR are selected from the first and the second experiments. Table 3 shows the results of training stage variation.

In most cases, classifiers with more stages achieved lower "False" detection. At the same time, classifiers with more stages provided more result in the "Miss" detection category as well. Classifiers with many states can have an overfitting model problem. An overfitting model generally occurs when a model is excessively complex, such as having too many training cycles or parameters relative to the number of observations. The model begins to memorize training data rather than learning to generalize from trend. Therefore, its performance is good on the training examples, while the performance on unseen data becomes worse. There is no reliable method to select the classification that always works. Therefore, a target of the classification model is selected for the maximum reduction in "False" detection and minimum decrease in "Hit" detection (which are italicized bold in Table 3). Figure 8 shows some of the detection results of " $n$ " (Ko kai) hand posture from each number of stages.

After the target classification models have been selected, we implement a multiple cascades structure to classify different hand postures. In structure, all cascades are loaded into the system simultaneously, and each cascade is responsible for detecting a single hand posture. Rectangle detection for different labels is used to tell which hand posture is detected. Based on the experimental results, we found that this method is fast enough to run in real time when we load all trained cascaded classifiers at the same time. Confusion may occur between hand postures. For example, the " $n$ " (Ko Kai) hand posture and number " 2 " hand posture may be confused with each other. However, this confusion can be resolved by computing the correlation coefficient between the detection results, with a set of appropriate reference images of the hand postures. Then, we pick the matched hand posture by choosing the one that gives the maximum correlation 
TABLE 2: The performance of 21 trained classifiers (adjusting TPR).

\begin{tabular}{|c|c|c|c|c|c|c|c|c|c|}
\hline & \multicolumn{3}{|c|}{$\mathrm{TPR}=0.995$ (1st experiment) } & \multicolumn{3}{|c|}{$\mathrm{TPR}=0.997$} & \multicolumn{3}{|c|}{$\mathrm{TPR}=0.999$} \\
\hline & Hit & Miss & False & Hit & Miss & False & Hit & Miss & False \\
\hline "1" & 45 & 5 & 198 & 45 & 5 & 305 & 46 & 4 & 247 \\
\hline "2" & 49 & 1 & 138 & 49 & 1 & 129 & 48 & 2 & 102 \\
\hline " $3 "$ & 49 & 1 & 58 & 49 & 1 & 59 & 49 & 1 & 32 \\
\hline “4” & 41 & 9 & 51 & 32 & 18 & 16 & 41 & 9 & 24 \\
\hline “5” & 48 & 2 & 91 & 45 & 5 & 49 & 47 & 3 & 62 \\
\hline “อ” & 50 & 0 & 69 & 49 & 1 & 87 & 49 & 1 & 77 \\
\hline "บ" & 42 & 8 & 276 & 28 & 22 & 295 & 35 & 15 & 197 \\
\hline “ด” & 48 & 2 & 42 & 43 & 7 & 86 & 43 & 7 & 69 \\
\hline “ฟ” & 44 & 6 & 164 & 41 & 9 & 124 & 37 & 13 & 98 \\
\hline “ห" & 33 & 17 & 376 & 22 & 28 & 351 & 29 & 21 & 361 \\
\hline “จ" & 44 & 6 & 114 & 44 & 6 & 118 & 45 & 5 & 95 \\
\hline “ $\cap "$ & 47 & 3 & 34 & 47 & 3 & 21 & 46 & 4 & 79 \\
\hline “ล" & 47 & 3 & 0 & 46 & 4 & 0 & 43 & 7 & 1 \\
\hline “ม" & 40 & 10 & 276 & 43 & 7 & 194 & 40 & 10 & 218 \\
\hline "น" & 45 & 5 & 301 & 45 & 5 & 159 & 37 & 13 & 418 \\
\hline “พ” & 46 & 4 & 153 & 46 & 4 & 178 & 41 & 9 & 167 \\
\hline "ร" & 44 & 6 & 54 & 46 & 4 & 83 & 44 & 6 & 60 \\
\hline “ส" & 49 & 1 & 6 & 50 & 0 & 22 & 50 & 0 & 29 \\
\hline “ต” & 47 & 3 & 66 & 45 & 5 & 65 & 47 & 3 & 42 \\
\hline “ว” & 40 & 10 & 278 & 36 & 14 & 469 & 30 & 20 & 467 \\
\hline “ย" & 46 & 4 & 39 & 46 & 4 & 73 & 44 & 6 & 107 \\
\hline
\end{tabular}

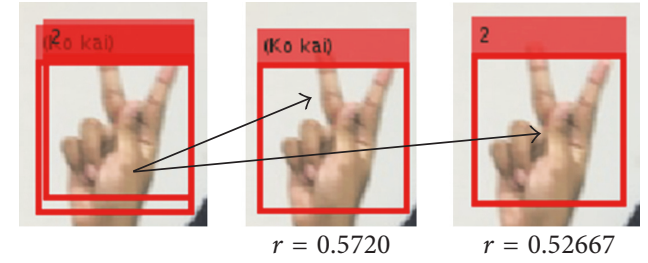

FIGURE 9: Correlation coefficient.

coefficient. We computed the correlation coefficient $(r)$ as follows:

$$
r=\frac{\sum_{m} \sum_{n}\left(A_{m n}-\bar{A}\right)\left(B_{m n}-\bar{B}\right)}{\sqrt{\sum_{m} \sum_{n}\left(A_{m n}-\bar{A}\right)^{2} \sum_{m} \sum_{n}\left(B_{m n}-\bar{B}\right)^{2}}} .
$$

Here $A$ and $B$ are images of the same size. $\bar{A}$ and $\bar{B}$ are the means of image elements. An example of the correlation coefficient of the " $n$ " (Ko kai) hand posture and number "2" hand posture is shown in Figure 9, with correlation coefficients of 0.5720 and 0.5267 , respectively.

Table 4 gives the confusion matrix for the detection of all hand posture classes, using a combination of all cascades, which were tested with 50 test images for each hand posture class. Rows are targets and sum up to one and columns are predictions. This shows confusion between similar-looking hand postures such as " $\mathrm{n}$ " (Ko kai) confused with " 2 " (6\%) and also “ล" (Lo ling), “ม” (Mo ma), and “น" (No nu). By analyzing the detection results, we found that some of the

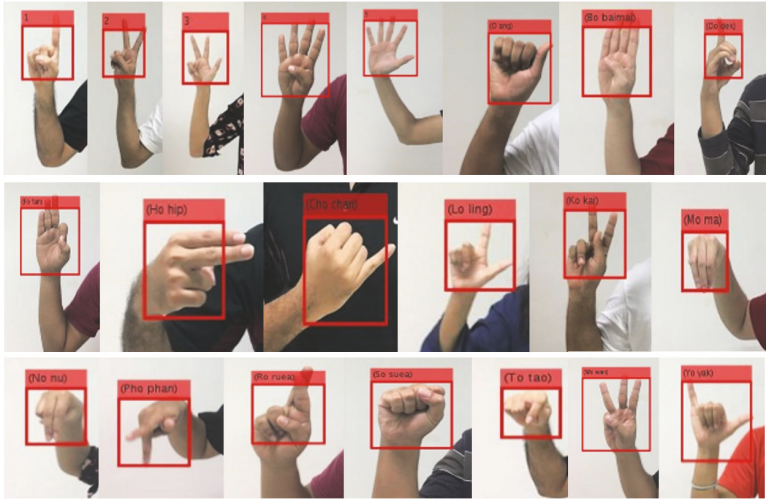

FIgURE 10: All hand postures detection.

"Miss" detections are caused by the excessive in-plane or outof-plane rotations due to hand posture variations and fingerspelling styles of different users. For the "False" detection, we found that the classification error might have occurred because there are some hand postures in Thai finger-spelling which are similar. For example, hand postures of "a" (O ang), “ม" (Mo ma), and “ส" (So suea) are all represented by a closed fist but differ only in the thumb position (depending on subject's dexterity), leading to higher confusion levels. Besides, the majority of "False" detections happened in small image areas. However, these small false detection boxes can be easily eliminated by defining a threshold for rectangular size. All hand postures detection is shown in Figure 10. 


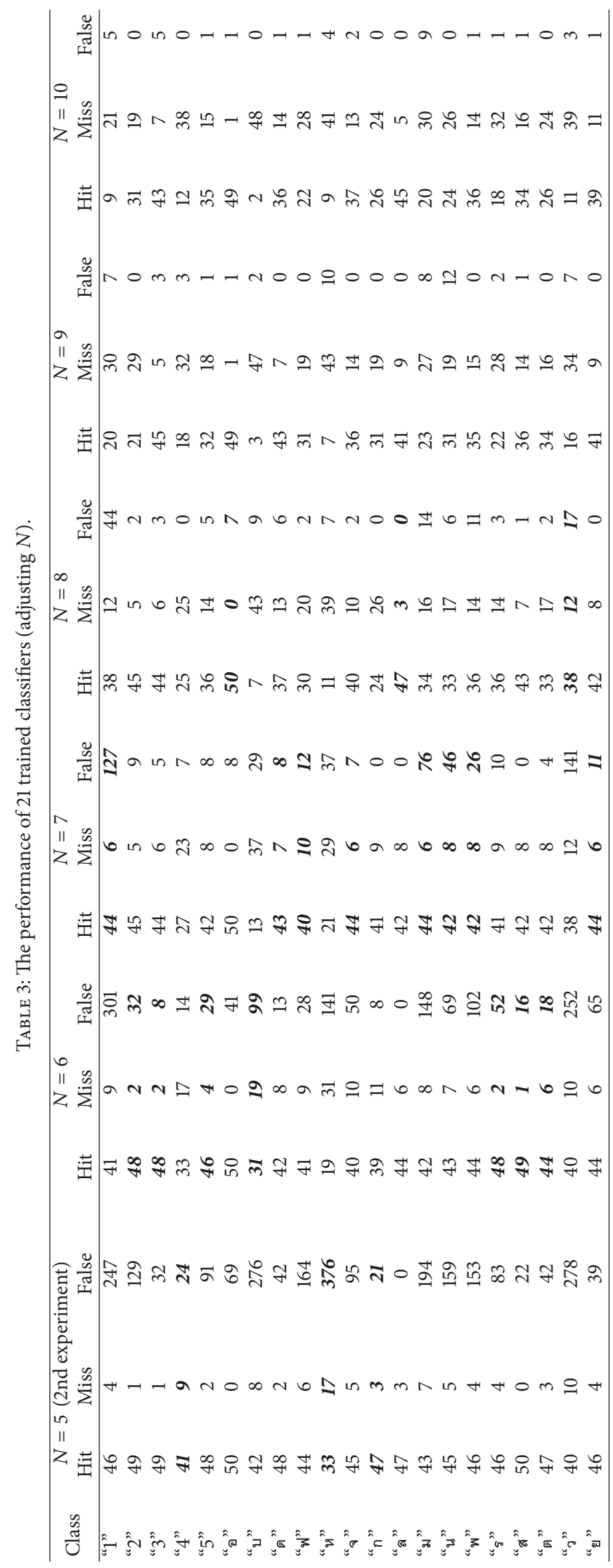




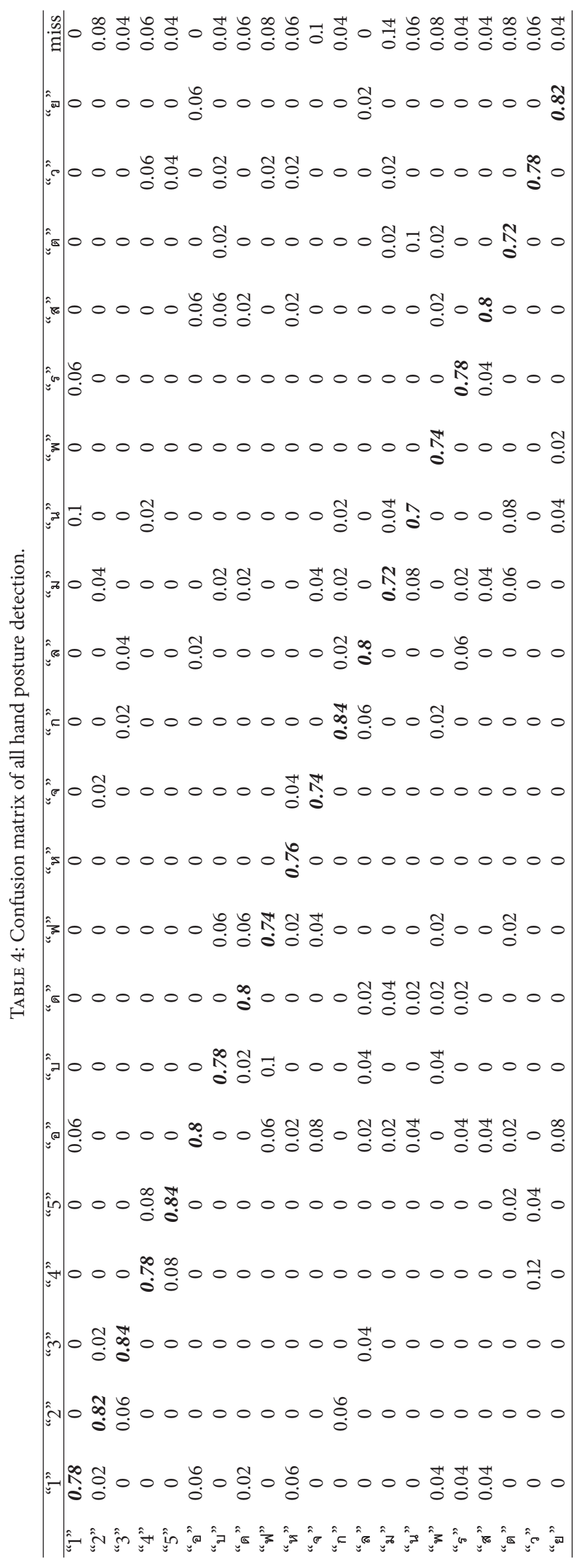


TABLE 5: General comparison with existing systems.

\begin{tabular}{|c|c|c|c|c|c|c|}
\hline Work & Device & Background & Outfit & Real time & Letters & Recognition rate \\
\hline Saengsri et al. [11] & Sensor glove & No & No & Yes & 16 & $94.44 \%$ \\
\hline Kanjanapatmata [12] & No & Yes & Yes & No & 15 & $72 \%$ \\
\hline Veerasakulthong [16] & Color glove & Yes & Yes & No & 31 & $88.26 \%$ \\
\hline Sriboonruang et al. [14] & No & Yes & Yes & No & $\mathrm{N} / \mathrm{A}^{*}$ & $72 \%$ \\
\hline Sakulsujirapa et al. [13] & No & Yes & Yes & No & 42 & $81.43 \%$ \\
\hline Phitakwinai et al. [15] & No & Yes & Yes & No & 15 & $79.90 \%$ \\
\hline Our method & No & No & No & Yes & 21 & $78 \%$ \\
\hline
\end{tabular}

${ }^{*} \mathrm{~N} / \mathrm{A}$ : not available.

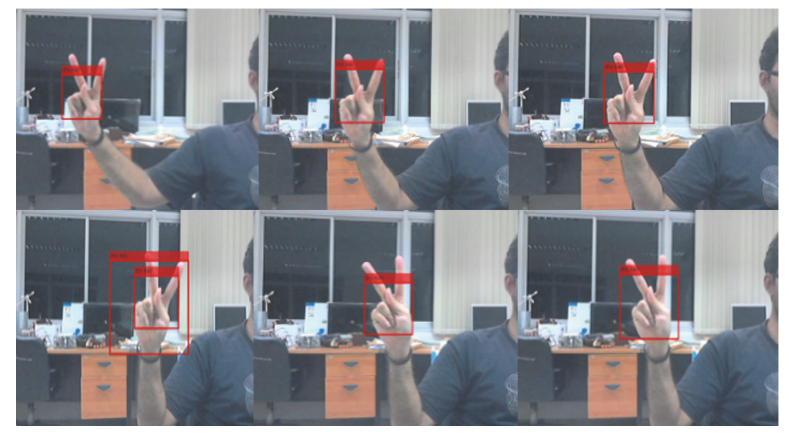

FIGURE 11: Real-time detection for the " $n$ " (Ko kai) hand posture.

To give general comparison between previous methods and our proposed method, some existing research works involving Thai finger-spelling recognition are shown in Table 5. We compared the general conditions not only for our method but also for some previous research that used other additional devices such as a sensor glove or a color glove. Regarding the background of the image, some researchers set background to a constant color. In terms of the outfit, users are asked to wear long-sleeves shirts. Concerning the number of the letters that can be recognized in the system, our system is not as good as the method that used additional devices such as glove based method because the image is not as good as a signal from an electronic sensor, especially when fingers occlude or stick together. For methods that use only camera images, it is also hard to compare recognition performance achieved from different datasets for testing in Thai fingerspelling. For the recognition rate, our average classification precision is around $78 \%$ for 21 hand postures classification. Although our work does not yield a more significant result compared to other techniques, by analyzing other conditions (see Table 5), they need to set background such as black or white color, additional device is required such as color glove to separate hand from other parts of body, and most of existing works cannot run in real time, while our system does not need to do any preprocessing or segmentation before computing the finger-spelling recognition and is fast enough to be run in real-time situation and clutter background as shown in Figure 11.

\section{Conclusion}

We proposed an approach to recognize hand posture in real time with a single web camera as the input device. The approach focused on hand posture recognition with Histogram of Orientation Gradient (HOG) and the AdaBoost learning algorithm. The Histogram of Orientation Gradient feature can effectively describe the hand posture pattern with a computation of gradient orientation. This feature allows the system to be able to distinguish the hand postures that are similar. The AdaBoost learning algorithm can greatly speed up detection performance and construct a strong classifier by combining a sequence of weak classifiers. The experimental results were tested by adjusting training parameters, false positive rates (FPR), true positive rates (TPR), and number of training stages $(N)$, to achieve the best classifier. A target of the classification model is selected for the maximum reduction in false detection and minimum decrease in detection. Based on the cascaded classifier, a multiple cascaded structure was implemented to classify different hand postures. The correlation coefficient must be computed when hand postures confuse each other. From experimental result, we found that the average classification accuracy is around $78 \%$. For work comparison, our method does not need to do any preprocessing or segmentation before computing the fingerspelling recognition and is fast enough to be run in real time. Furthermore, this method can be used with other problems in object detection field such as human, car, or symbol detector. In future work, we will implement the sequence recognition for other letters in Thai finger-spelling. Some letters in Thai finger-spelling occur from combination of hand posture. For example, " $n$ " (Ko kai) combined with digit "1." This sequence of hand posture will be translated to " $v$ " (Kho Khai). For sequence recognition, a Finite State Machine (FSM) or a Hidden Markov Model (HMM) can be used to define the rule for the recognition process. Furthermore, if information of finger was taken into account and trained with more dataset images, then the errors of the classifier should be reduced.

\section{Conflicts of Interest}

The author declares that there are no conflicts of interest regarding the publication of this paper. 


\section{Acknowledgments}

This work was supported by the Higher Education Research Promotion and National Research University Project of Thailand, Office of the Higher Education Commission.

\section{References}

[1] T. B. Dinh, V. B. Dang, D. A. Duong, T. T. Nguyen, and D.-D. Le, "Hand gesture classification using boosted cascade of classifiers," in Proceedings of the 4th IEEE International Conference on Research, Innovation and Vision for the Future, RIVF'06, pp. 139-144, vnm, February 2006.

[2] R. Feris, M. Turk, R. Raskar, K. Tan, and G. Ohashi, "Exploiting depth discontinuities for vision-based fingerspelling recognition," in Proceedings of the 2004 IEEE Computer Society Conference on Computer Vision and Pattern Recognition Workshops, CVPRW 2004, usa, July 2004.

[3] S. Ricco and C. Tomasi, "Fingerspelling recognition through classification of letter-to-letter transitions," Lecture Notes in Computer Science (including subseries Lecture Notes in Artificial Intelligence and Lecture Notes in Bioinformatics), vol. 5996, no. 3, pp. 214-225, 2010.

[4] Z. Mo and U. Neumann, "Real-time hand pose recognition using low-resolution depth images," in Proceedings of the 2006 IEEE Computer Society Conference on Computer Vision and Pattern Recognition, CVPR 2006, pp. 1499-1505, usa, June 2006.

[5] P. Goh and E.-J. Holden, "Dynamic fingerspelling recognition using geometric and motion features," in Proceedings of the 2006 IEEE International Conference on Image Processing, ICIP '06, pp. 2741-2744, October 2006.

[6] S. Liwicki and M. Everingham, "Automatic recognition of fingerspelled words in british sign language," in Proceedings of the 2009 IEEE Conference on Computer Vision and Pattern Recognition, CVPR 2009, pp. 50-57, usa, June 2009.

[7] W. Jiangqin and G. Wen, "The recognition of finger-spelling for chinese sign language," in Proceedings of the International Gesture Workshop on Gesture and Sign Languages in HumanComputer Interaction, vol. 2001, 2005.

[8] X. Teng, B. Wu, W. Yu, and C. Liu, "A hand gesture recognition system based on local linear embedding," Journal of Visual Languages Computing Volume 16, vol. Issue 5, pp. 442-454, 2005.

[9] K. Fujimura and X. Liu, "Sign recognition using depth image streams," in Proceedings of the FGR 2006: 7th International Conference on Automatic Face and Gesture Recognition, pp. 381386, April 2006.

[10] Y. Tabata and T. Kuroda, "Finger spelling recognition using distinctive features of hand shape in," 7th ICDVRAT with Art Abilitation, pp. 287-292, 2008.

[11] S. Saengsri, V. Niennattrakul, and C. A. Ratanamahatana, "TFRS: thai finger-spelling sign language recognition system," in Proceedings of the 2012 2nd International Conference on Digital Information and Communication Technology and its Applications, DICTAP 2012, pp. 457-462, tha, May 2012.

[12] W. Kanjanapatmata, Sign language gesture recognition for Thai consonant using artificial neural Networks, thesis, King Mongkut's University of Technology North Bangkok, Bangkok, Thailand, 2005.
[13] B. Sakulsujirapa, M. Karnjanadecha, and A. Choksuriwong, "Thai sign language recognition based on finger pattern analysis," in Proceedings of the On Embedded Systems and Intelligent Technology, 16 pages, 2011.

[14] Y. Sriboonruang, P. Kumhom, and K. Chamnongthai, "Hand posture classification using wavelet moment invariant," in Proceedings of the 2004 IEEE Symposium on Virtual Environments, Human-Computer Interfaces and Measurement Systems, VECIMS, pp. 78-82, usa, July 2004.

[15] S. Phitakwinai, S. Auephanwiriyakul, and N. Theera-Umpon, "Thai sign language translation using Fuzzy C-Means and scale invariant feature transform," Lecture Notes in Computer Science (including subseries Lecture Notes in Artificial Intelligence and Lecture Notes in Bioinformatics), vol. 5073, no. 2, pp. 1107-1119, 2008.

[16] W. Veerasakulthong, Thai hand sign recognition [M.S. thesis], National Institute of Development Administration, Bangkok, Thailand, 2007.

[17] K. Silanon, "Thai finger-spelling computer-assisted instruction for hearing and speaking," in Proceedings of the 10th international Convention on Rehabilitation Engineering Assistive Technology, p2.2, 2016.

[18] N. Dalal and B. Triggs, "Histograms of oriented gradients for human detection," in Proceedings of the IEEE Computer Society Conference on Computer Vision and Pattern Recognition (CVPR '05), vol. 1, pp. 886-893, June 2005.

[19] W. Li, Y. Lin, B. Fu, M. Sun, and W. Wu, "Cascade classifier using combination of histograms of oriented gradients for rapid pedestrian detection," Journal of Software, vol. 8, no. 1, pp. 71-77, 2013.

[20] H. Liu, T. Xu, X. Wang, and Y. Qian, "Related HOG features for human detection using cascaded adaboost and SVM classifiers," Lecture Notes in Computer Science (including subseries Lecture Notes in Artificial Intelligence and Lecture Notes in Bioinformatics), vol. 7733, no. 2, pp. 345-355, 2013.

[21] Q. Zhu, S. Avidan, M.-C. Yeh, and K.-T. Cheng, "Fast human detection using a cascade of histograms of oriented gradients," in Proceedings of the IEEE Computer Society Conference on Computer Vision and Pattern Recognition (CVPR '06), vol. 2, pp. 1491-1498, IEEE, June 2006.

[22] P. Negri, X. Clady, and L. Prevost, "Benchmarking haar and histograms of oriented gradients features applied to vehicle detection," in Proceedings of the 4th International Conference on Informatics in Control, Automation and Robotics, ICINCO 2007, pp. 359-364, fra, May 2007.

[23] Q. Chen and N. D. Georganas, "Hand gesture recognition using haar-like features and a stochastic context-free," IEEE Transactions on Instrumentation and Measurement, vol. 57, no. 8, 2008

[24] S.-K. Pavani, D. Delgado, and A. F. Frangi, "Haar-like features with optimally weighted rectangles for rapid object detection," Pattern Recognition, vol. 43, no. 1, pp. 160-172, 2010.

[25] P. Viola and M. Jones, "Rapid object detection using a boosted cascade of simple features," in Proceedings of the IEEE Computer Society Conference on Computer Vision and Pattern Recognition, pp. I511-I518, December 2001. 

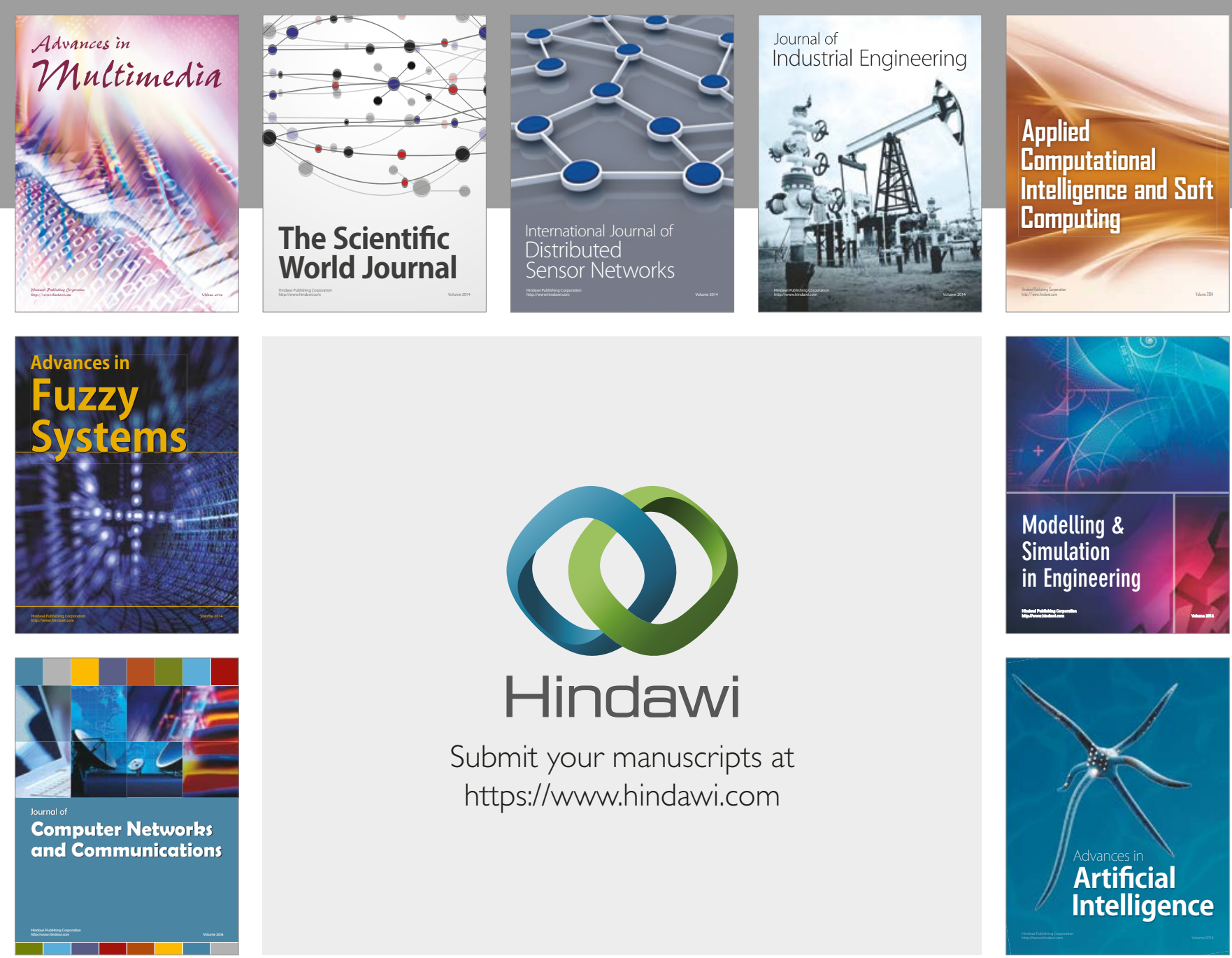

\section{Hindawi}

Submit your manuscripts at

https://www.hindawi.com
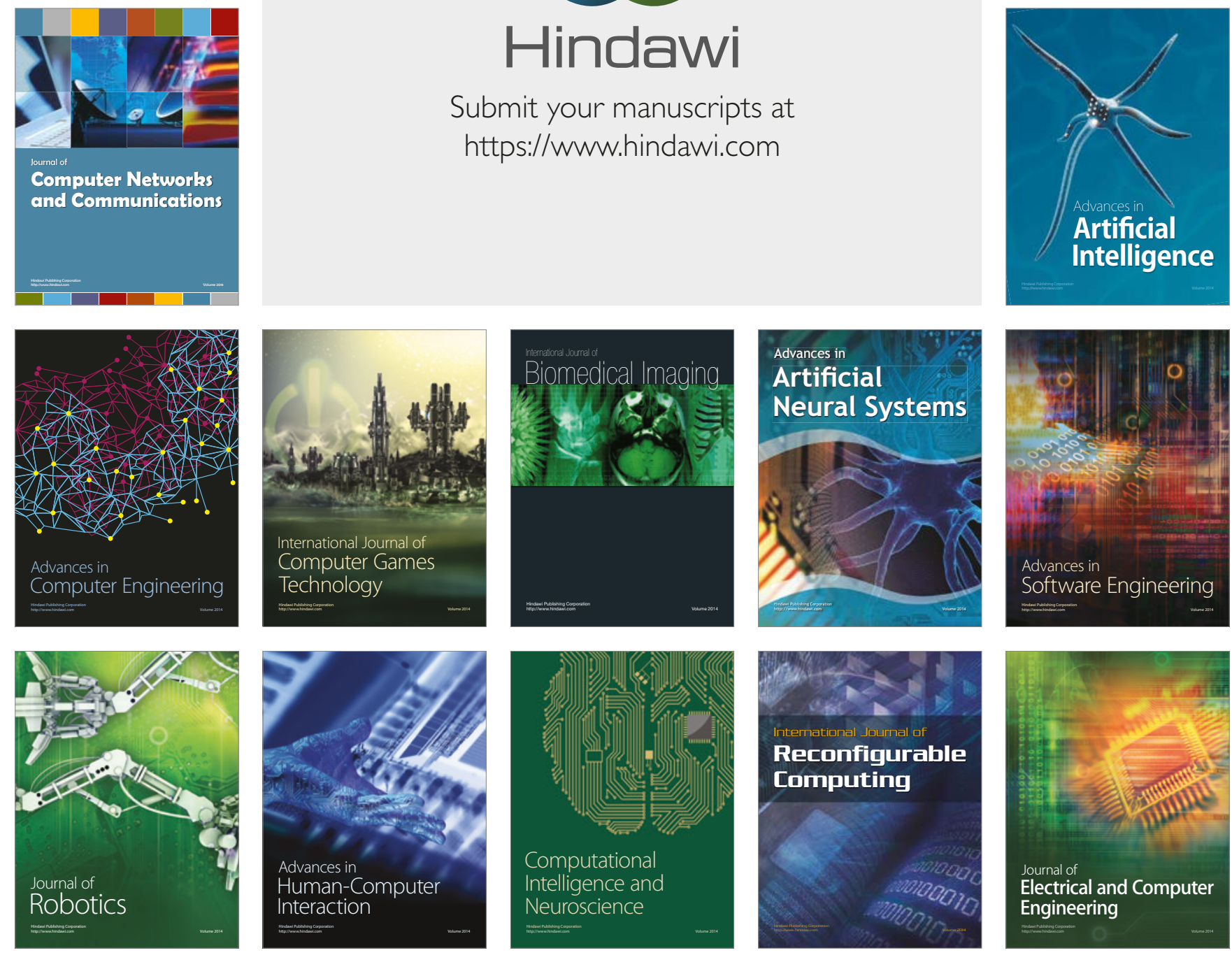